\title{
Finite Element Analysis and Parameter Influence of Non-developable Ruled Surface Impeller Blade in Flank Milling
}

\author{
Yu Sun, Lixin Cao \\ School of Mechanical Engineering, Dalian University of Technology, Dalian, China
}

Email address:

jtreasure@qq.com (Yu Sun), caosm@dlut.edu.cn (Lixin Cao)

To cite this article:

Yu Sun, Lixin Cao. Finite Element Analysis and Parameter Influence of Non-developable Ruled Surface Impeller Blade in Flank Milling. Science Discovery. Vol. 8, No. 1, 2020, pp. 12-17. doi: 10.11648/j.sd.20200801.14

Received: April 3, 2020; Accepted: May 12, 2020; Published: May 19, 2020

\begin{abstract}
Through the cutting simulation of titanium alloy impeller blade, the effect of the actual milling processing path on the elastic part deflection to of complicated thin-walled workpiece with low rigidity and high precision is studied. Use the principle of impeller modeling to model the spline of the impeller, and the spline curve of the impeller generated in MABLEB is imported into UG for digital modeling. The spline curve is used to generate the neutral surface, and the suction surface and pressure surface are obtained by the method of non-equidistant offset. The model of the non-stretchable straight surface blade is imported into ABAQUS for static simulation. Using MATLAB to fit the experimental data in the reference literature that fits the milling process range, the side milling experience formula is fitted. Calculate the equivalent milling force corresponding to different axial cutting depths, import into ABAQUS and use Python for secondary development. By simulating the milling process through the life-and-death element method, the local elastic deformation law of the milled thin-walled parts when multi-layer milling the impeller blades is explored. The influence of different machining paths on the deformation of the tool is studied. Simulation results show that the part deflection of workpiece can be reduced by rationally planning the machining path.
\end{abstract}

Keywords: Titanium Alloy Impeller Blade, Cutting Simulation, Processing Path, Part Deflection

\section{非可展直纹面叶轮叶片侧铣加工变形的有限元分析及参数影响}

\section{孙瑜, 曹利新}

大连理工大学, 机械工程学院, 大连, 中国

\section{邮箱}

jtreasure@qq.com（孙瑜）, caosm@dlut.edu.cn（曹利新）

摘要: 通过对钛合金叶轮叶片进行切削仿真, 研究实际铣削加工路径对低刚度高精度复杂薄壁件的弹性让刀变形的影 响。利用叶轮建模原理, 对叶轮的样条进行造型, 将MATLEB中生成的叶轮的样条曲线导入UG中进行数字化建模。 利用样条曲线生成中性面, 通过非等距离偏置的原理得到吸力面和压力面的造型, 将建立的非可展直纹面叶片模型导 入ABAQUS中, 对其进行静态仿真。利用MATLAB对参考文献中符合铣削加工范围的实验所得数据进行立铣刀侧铣加 工经验公式的拟合。计算出不同轴向切深对应的等效铣削力, 导入ABAQUS中利用Python进行二次开发, 通过生死单 元法模拟铣削过程, 探究对叶轮叶片进行多层铣削时铣削薄壁件的局部弹性变形规律。研究不同加工路径对让刀变形 的影响，仿真结果证明通过合理规划加工路径可以减小工件让刀变形。 
关键词: 钛合金叶轮叶片, 切削仿真, 加工路径, 让刀变形

\section{1. 引言}

薄壁件在铣削加工的过程中，由于其刚度低，易发生加 工变形, 导致被加工表面尺寸精度低, 影响工件性能[1]。钣 合金硬度远远高于铝合金, 常温下弹性模量约为铝合金的 1.5 倍, 刚度和强度都很大, 所以其弹性变形和塑性变形都相对 于铝合金要小, 密度仅为钢的 $60 \%$, 因此广泛适用于航空类 结构件的制造, 是一种典型的难加工材料。大量的文献对薄 壁件的弹性让刀变形、装夹变形和残余应力变形进行研究。 罗宇[2]等人研究了一种铝合金大型薄壁桶件, 通过对材料进 行热力耦合动态仿真, 得到切削热和切削力的变化规律, 通 过薄壁件的热处理得到初始残余应力, 将初始残余应力、切 削力和装夹条件进行耦合, 得到工件的残余应力分布和加工 变形情况。钱丽丽[3]等人研究了钛合金薄壁联动环, 分析了 联动环的工艺路线, 建立立铣刀的铣削力经验模型, 通过耦 合热处理后的残余应力、铣削切断力和装夹, 分别得出刀和 工件的变形图, 并对ABAQUS进行二次开发做参数化建模。 赵肖[4]等人利用薄板弹塑性力学原理求解薄壁件“S”件不同 加工位置处的基于精确铣削力模型的弹性变形, 并进行铣削 加工刀位轨迹优化, 借助 Matlab 建立理论补偿模型, 提高 了“S”件表面加工精度。S. Ratchev[5-9]等人建立多种集成方 法, 用于多步仿真迭代、建模和预测低刚度零件挠度引起的 表面误差、分析柔性力模型引起的变形、多级加工误差预测 及补偿方法。王荣奇 $[10]$ 等人探究了不同装夹方式和不同走 刀路径排列组合对薄板件的弹性变形影响, 并分析了未铣削 区域的材料残留对整体刚度带来的影响, 证明材料残留不仅 导致弹性变形的分布变化,还会使整体变形变小。袁俊松 [11] 等人再假定简单薄壁件模型铣削力恒定不变的基础上, 以 移动载荷的方式将等效铣削力动态加载在不同的刀位点 上观察其弹性变形, 不考虑刚度变化带来的影响。

对于非可展直纹面叶轮的研究也层出不穷。田燕宾、于 海滨[12-13]等利用UG建立半开式叶轮模型, 利用铣削力正交 试验建立了铣削力经验公式, 利用ANSYS通过直接在已加工 表面加载静态载荷的方式对叶片进行加工弹性变形分析, 并 对铣削加工参数进行遗传算法优化。刘永峰[14]等利用点铣法 加工叶片, 观察弹性让刀变形的同时优化加工余量, 基于刀 具运动约束的前提下将叶片上的刀路轨迹转换成局部坐标幅 值, 利用动态仿真模拟刀具曲线运动。Shi Wu[15]等人提出并 构造了机床切削工具的整体刚度和工件刚度分布的系统刚度 场, 通过运用多体变形理论设置采样点, 通过不同的工具空 间手势来开发处理系统的集成刚度场, 利用实验得出结论叶 片顶部的刚度较弱, 具有强烈的振动和较低的加工精度。黄 泽华 [16]对叶轮进行多轴变工况条件下铣削, 对弹性变形进行 误差补偿。

目前对于叶轮侧铣加工的有限元分析多采用直接载荷添 加的方法, 这种方法假设待加工表面已去除, 直接添加等效 铣削力。直接添加法所得的让刀变形仅仅为弹性伸长量不同 所导致的刚度减弱带来的变形。这种方法没有整体薄壁件刚 度上的变化, 铣削加工顺序对其没有影响。在研究整体刚度
对薄壁件侧铣加工带来影响的文献中多采用规则结构件, 规 则结构件的网格为形状大小一致的六面体网格, 所以只需要 得到一个力即可为每一个网格添加不变的等效铣削力。由于 叶轮叶片是一个典型的非可展直纹面, 因此在进行生死单元 法时需要更多的时间去构造网格, 且每一个网格形状大小不 一致, 所以需要构造经验公式并利用ABAQUS的二次开发在 每一个网格上添加不同的铣削力。因此本文开展相应的仿真 铁削加工对复杂结构件的总体刚度变化带来的局部让刀变形 具有参考意义。本文在UG中的对叶片进行建模, 并将模型导 入ABAQUS中, 分层进行侧铣加工。运用等效切削力方法, 采用Python语言简化ABAQUS建模操作, 最终得出侧铣让刀 变形。

\section{2. 叶片建模}

叶片建模首先需要利用轴盘线和盖盘线来构建一个 非可展直纹面性质的中性面。坐标点通过利用非均匀有理 $B$ 样条进行拟合得到轴盘线和盖盘线，其定义如下:

$$
p(u)=\sum_{i=0}^{n} d_{i} N_{i, k}(u)
$$

其中 $\mathrm{u}$ 为节点值, $d_{i}$ 为控制顶点, $\mathrm{n}$ 为 $d_{i}$ 的个数, $\mathrm{k}$ 为 样条阶次, 此处取 $\mathrm{k}=3, N_{i, k}$ 为基函数, 可由de Boor-Cox 递推公式推得:

$$
\left\{\begin{array}{l}
N_{i, 0}(u)= \begin{cases}1, & u_{i}<u<u_{i+1} \\
0, & \text { 其他 }\end{cases} \\
N_{i, k}(u)=\frac{u-u_{i}}{u_{i+k}-u_{i}} N_{i, k-1}(u)+\frac{u_{i+k+1}-u}{u_{i+k+1}-u_{i+1}} N_{i+1, k-1}(u) \\
\text { 规定 } \frac{0}{0}=0
\end{array}\right.
$$

由定义公式(1)和公式(2)可知定义一条B样条需要 $\mathrm{n}+1$ 个控制顶点和 $\mathrm{n}+\mathrm{k}+2$ 个节点值, 设节点矢量为 $U\left(u_{0}, u_{1}, \ldots u_{i+k+1}\right)$ 。通过实验测得的 $\mathrm{m}+1$ 个坐标点设为 $P_{u}(u=0,1, \ldots, m)$, 要想求控制点, 首先要求节点矢量的值。 对于 $\mathrm{k}=3$ 而言, 累计弦长法是利用控制顶点连成的多边形 拉直成一条线段, 从起点到控制顶点的线段长度相对于总 长度的占比就是节点值的大小。通过累计弦长参数法可以 得到 $\mathrm{m}+1$ 个节点值, 公式如下:

$$
\left\{\begin{array}{l}
a_{o}=0 \\
a_{i}=a_{i-1}+\frac{L_{i-1}}{\sum_{i=1}^{m-1} L} \quad i=1,2, \ldots m-1 \\
a_{m}=1
\end{array}\right.
$$

其中 $L_{i-1}=\left|d_{i}-d_{i-1}\right|$, 为控制多边形边长, 利用式(3) 可得 $m+1$ 个节点值。由于 $k$ 次样条上的一个节点的位置与 
$k+1$ 个控制顶点有关, 所以为了使样条端点满足贝奇尔曲 线的性质，在第一个节点和最后一个节点分别多添加 $\mathrm{k}$ 个 重复节点值。则节点矢量为:

$$
U=\left[0,0,0,0, a_{1}, a_{2}, \ldots a_{m-1}, 1,1,1,1\right]
$$

由 $n+k+2=m+1+2 k$ 可知若要得到 $\mathrm{n}+1$ 个控制顶点 需要 $n+1=m+k=m+3$ 个坐标点建立方程, 而坐标点只 有 $\mathrm{m}+1$ 个:

$$
P_{i}=p(u)=N_{i, 3}\left(u_{i}\right) d_{i}+N_{i+1,3}\left(u_{i}\right) d_{i+1}+N_{i+2,3}\left(u_{i}\right) d_{i+2}
$$

因此利用切矢边界条件:

$$
\left\{\begin{array}{l}
d_{1}-d_{0}=\frac{a_{1}}{3} \dot{P}_{0} \\
d_{n}-d_{n-1}=\frac{1-a_{m-1}}{3} \dot{P}_{n}
\end{array}\right.
$$

将(5)和(6)联立可解得控制顶点, 用MATLAB画出样 条曲线, 并将数据导入UG中。将轴盘曲线和盖盘曲线利 用直线进行连接成封闭环, 利用直纹面命令生成中性面, 中性面是一个非可展直纹面。

在UG中做位于样条上数据点处的直纹面的法向量, 此向量垂直于样条在数据点处的切向量与过数据点的一 条母线所构成的平面。沿法向量的正负方向分别偏置叶片 厚度的一半, 由于每个数据点处叶片厚度不同, 因此由偏 置过的数据点插值形成的样条曲线是中性面上曲线的变 距偏置曲线。用直线将偏置后的曲线进行连接, 利用直纹 面命令生成吸力面和压力面, 利用相同方法扫描出其他连 接面, 利用片体缝合命令生成实体叶片。由此可得吸力面 和压力面也是非可展直纹面, 叶片构造如下:

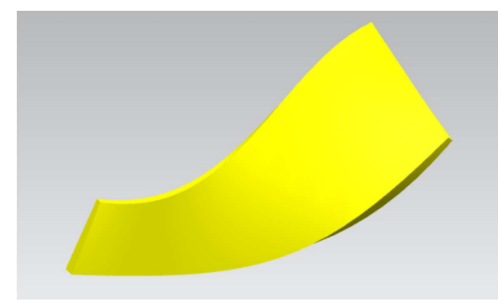

图1 叶轮叶片模型。

\section{3. 切削力经验公式的拟合}

本文采用侧铣加工的方式对叶轮叶片进行仿真, 并采 用分层铣削的加工方法。由上文建模可知, 吸力面和压力 面都是非可展直纹面, 所以加工轨迹复杂, 采用动态铣削 的方式难以规划刀具路径。因此采用静态铣削的方法, 利 用等效集中力的方式, 添加到网格的节点上, 模拟加工过 程。

叶轮叶片进气边高, 出气边矮, 因此在分层加工过程 中, 不考虑已加工表面再次进行铣削的前提下, 每一层的 每一时刻, 刀具侧铣的轴向切深都有所不同, 导致铣削力 不同。这就导致侧铣叶片的仿真不同于薄壁多框类结构件 或薄壁桶件等规则件, 只要进行动态仿真微铣削, 得到固
定的支反力就可以进行添加。杨金龙在参考文献[17]中利 用立式加工中心对钛合金薄壁件进行铣削实验, 并对试验 中获得的总切削力和粗粘度进行了经验公式拟合, 并对模 型进行了验证。本文拟利用参考文献[17]中的实验数据, 利用MATLAB拟合切向铣削力、法向铣削力和轴向铣削力 的经验公式, 通过输入不同的侧铣参数达到添加不同铣削 力的目的。在选用钛合金的经验公式的过程中, 要注意选 择铣削参数范围相近的实验数据, 每一组经验公式只在选 定的参数范围内具有正确性。

钛合金具有高硬度的特点, 因此需要耐磨的硬质合金 刀具, 在此选用钨钴合金圆柱铣刀。设刀具前角 $10^{\circ}$, 后 角 $12^{\circ}$, 螺旋角 $38^{\circ}$, 刀具齿数为 2 , 半径为 $3 \mathrm{~mm}$ 。设铣刀 侧铣加工的经验公式为:

$$
F=C_{F} a_{p}^{b_{1}} a_{e}^{b_{2}} f_{z}^{b_{3}} v_{c}^{b_{4}}
$$

其中 $C_{F}$ 为系数, $a_{p}$ 为轴向切深, $a_{e}$ 为径向切深, $f_{z}$ 为每齿进给量, $v_{c}$ 为刀具旋转速度, $w, x, y, z$ 为各切削 参数的系数, 即切削参数对铣削力的影响因子。正交试验 数据如图2所示。将公式2-1两边取对数得:

$$
\ln F=\ln C_{F}+b_{1} \ln a_{p}+b_{2} \ln a_{e}+b_{3} \ln f_{Z}+b_{4} \ln v_{c}
$$

其中令

$$
\left\{\begin{array}{l}
y=\ln F, b_{0}=\ln C_{F} \\
x_{1}=\ln a_{p}, x_{2}=\ln a_{e} \\
x_{3}=\ln f_{Z}, x_{4}=\ln v_{c}
\end{array}\right.
$$

则 16 个试验的多元回归方程可写作:

$$
y=b_{0}+b_{1} x_{1}+b_{2} x_{2}+b_{3} x_{3}+b_{4} x_{4}
$$

则残差的方差 $\mathrm{Q}$ 为:

$$
Q=\sum_{k=1}^{16} \varepsilon_{k}^{2}=\sum_{k=1}^{16}\left[y_{k}-\left(b_{0}+b_{1} x_{k 1}+b_{2} x_{k 2}+b_{3} x_{k 3}+b_{4} x_{k 4}\right)\right]^{2}
$$

对等式两边求偏导, 利用常数求导为 0 得:

$$
\left\{\begin{array}{l}
\frac{\partial Q}{\partial b_{0}}=\frac{\partial Q}{\partial b_{i}}=0, \quad i=1,2,3,4 \\
\frac{\partial Q}{\partial b_{0}}=-2 \sum_{k=1}^{16}\left(y_{k}-b_{0}-b_{1} x_{k 1}-b_{2} x_{k 2}-b_{3} x_{k 3}-b_{4} x_{k 4}\right) \\
\frac{\partial Q}{\partial b_{i}}=-2 \sum_{k=1}^{16}\left(y_{k}-b_{0}-b_{1} x_{k 1}-b_{2} x_{k 2}-b_{3} x_{k 3}-b_{4} x_{k 4}\right) x_{k i}
\end{array}\right.
$$

由此可求得 $b_{0}, b_{1}, b_{2}, b_{3}, b_{4}$ 的大小。线性回归方程可由 MATLAB编程获得:

$$
\begin{aligned}
& F x=e^{-1.1353} a_{p}^{0.2943} a_{e}^{0.5543} f_{Z}^{-0.0605} v_{c}^{1.2139} \\
& F y=e^{-0.7684} a_{p}^{0.2943} a_{e}^{0.5352} f_{Z}^{-0.1044} v_{c}^{1.1157} \\
& F z=e^{-1.2098} a_{p}^{0.1542} a_{e}^{0.4620} f_{Z}^{0.1564} v_{c}^{1.2291}
\end{aligned}
$$


由残差图可知第二组试验的数据误差较大属于异常 点, 优化后残差图如图2所示, 可见误差在 $10 \%$ 以内。由 利用 $\mathrm{F}$ 检验法检验回归模型可得相关系数 $r^{2}=0.9708$ 十分 接近于 1 , 观测值 $F=91.5359$ 且 $F$ 的概率 $p=2 e^{-8}$ 远远小于显著 性水平 0.05 , 因此模型回归显著。

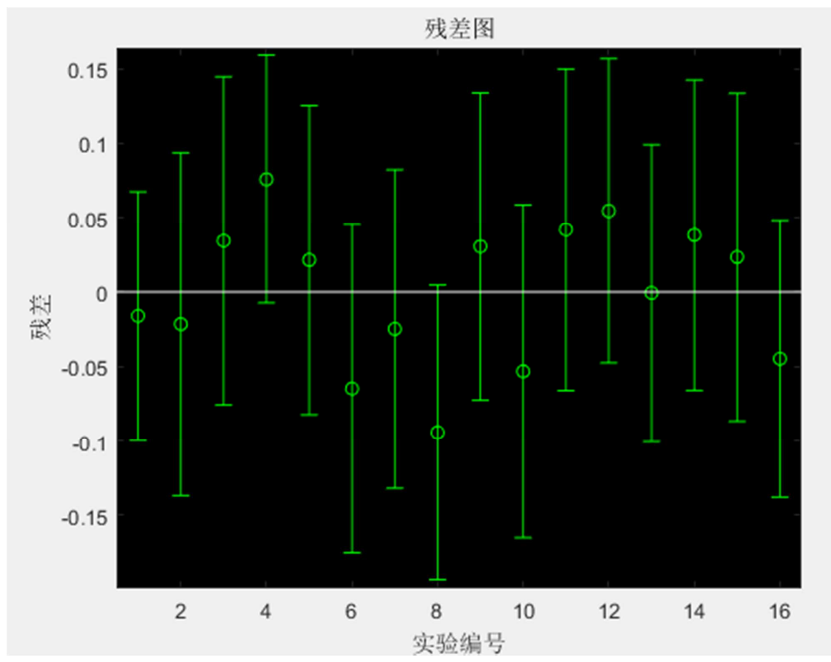

图2 残差图。

\section{4. 有限元仿真及结果分析}

\section{1. 有限元仿真}

将UG中建立的模型导入ABAQUS中，在薄壁件的加 工仿真过程中, 由于每个工步去除量较小, 塑性变形过小, 因此主要研究让刀变形。由文献[18]可知在铣削力不够大
的情况下, 初始残余应力的影响力远远大于铣削力的影响, 铣削力无法对工件产生整体的变形, 只是影响了局部变形。 设工件介质均匀且具有各向同性, 弹性模量 $\mathrm{E}=11.2 \mathrm{e}^{4} \mathrm{MPa}$, 泊松比 $\mu=0.34$, 密度为 $5.44 \mathrm{~T} / \mathrm{mm}^{2}$ 。

本文叶片最薄处仅为 $2 \mathrm{~mm}$, 压力面和吸力面的侧铣径 向切深对叶片总刚度产生很大影响, 当力达到铣削力的大 小时, 切屑离开工件。简单的载荷动态添加过程不足以表 达出刚度变化带来的让刀误差, 并且加工路径对叶片的刚 度不会产生影响进而对让刀产生影响。因此需要使用“生 死单元”的方法进行研究, 生死单元的死单元是用大小为 $1 \mathrm{e}^{-6}$ 的微小系数与单元的刚度矩阵相乘使其失效的过程。 单元“死”后其载荷、质量等材料性质趋近于 0 , 此单元的 应力应变不再影响其他单元, 使得总体刚度发生变化, 更 贴近于实际效果, 且加工路径对刚度变化将产生不同影响。 在顺铣加工过程中, 铣刀既自转, 又向坐标轴方向进给, 瞬时铣削厚度不断减小，在工件上的运动轨迹是次摆线， 公式为:

$$
\left\{\begin{array}{l}
x= \pm r \phi+R \sin \phi \\
y=R(1-\cos \phi)
\end{array}\right.
$$

为了便于力的施加, 假设待加工表面铣削厚度不变, 则将不规则表面以六面体网格形式呈现出来, 网格类型设 为C3D8R, 采用中性轴算法扫描技术添加网格, 使网格均 匀且序号连续。将铣削切削刃与工件瞬时接触线上的载荷 近似为在网格节点上施加集中力, 原理如图3所示, 网格 划分如图4所示。
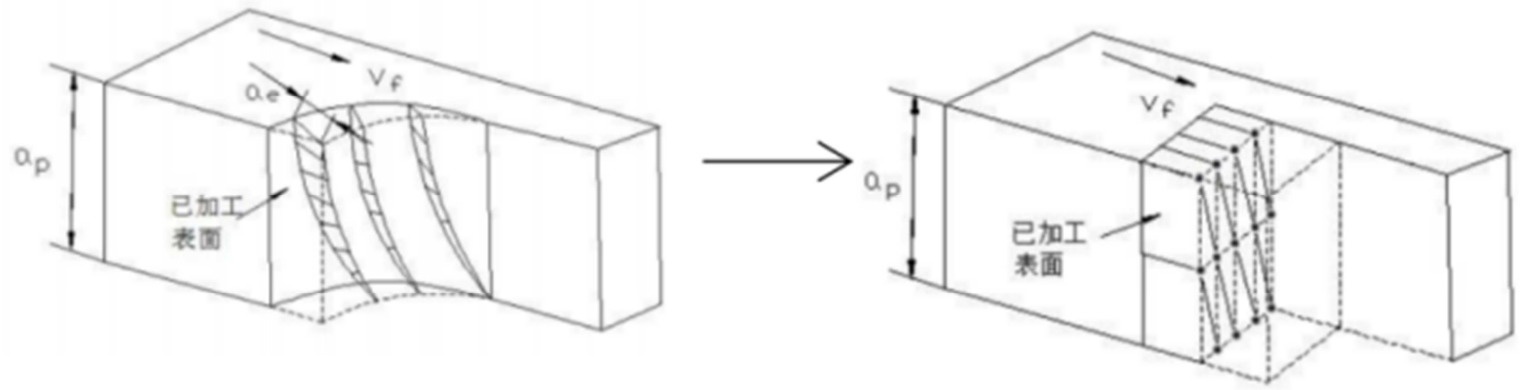

图3 力的添加。

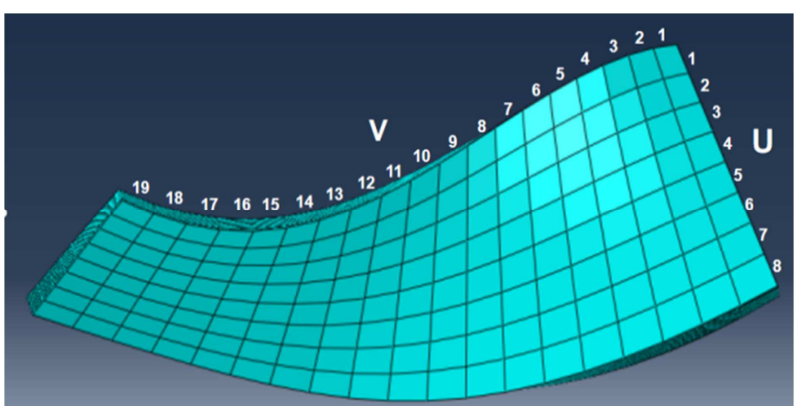

图4 网格划分。
设母线方向为铣削层数 $\mathrm{U}$, 样条方向为离散铣削点 $\mathrm{V}$, 吸力面为 $\mathrm{A}$ 面, 压力面为 $\mathrm{B}$ 面, 研究加工顺序对让刀变形 的影响。基于表面加工质量优先的前提下，叶片的所有面 皆采用顺铣进行铣削, 因此往复铣削回程过程中由于回程 运动是逆铣而不做考虑, 本文研究单向铣削和螺旋铣削两 种铣削方式。单向铣削先将A面沿U铣削8层至吸力面全部 完成, 再进行 $\mathrm{B}$ 面的8层铣削加工至压力面完成, 铣削方向 皆为V1到V19方向, 使得每一面都是先铣高点再铣低点, 铣削刀路轨迹如图 5 所示。在单向铣削时, 每加工一层都 要进行快速非铣削移刀, 换面的过程中需要由右旋刀换左 旋刀。 


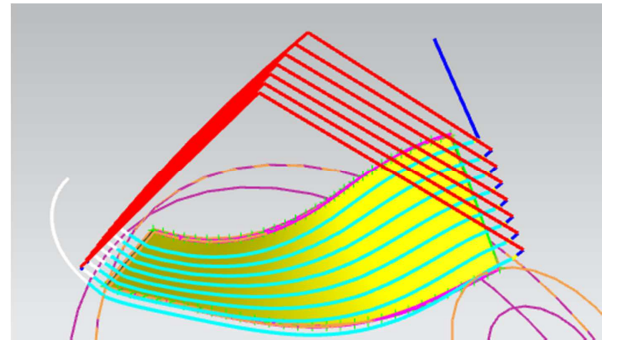

图5 单向铣削刀路。

螺旋铣削则将A面U1层铣削完成后铣削B面的U1层, 再铣削 $\mathrm{A}$ 面的 $\mathrm{U} 2$ 层, 以此类推, 螺旋铣削在 $\mathrm{A}$ 面方向为 $\mathrm{V} 1$ 到 $\mathrm{V} 19$ 方向, 在 $\mathrm{B}$ 面则为 $\mathrm{V} 19$ 到 $\mathrm{V} 1$ 向, 使得叶片每次都是 铣削最上层, 铣削刀路轨迹如图6所示。

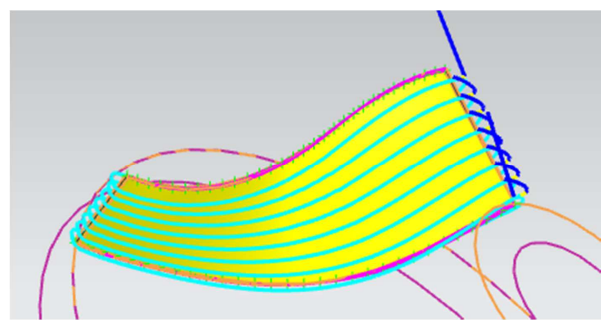

图6 螺旋铣削刀路。

侧铣加工的过程中按照实际铣削加工顺序, 先在网格 节点上施加集中力, 随后网格消失, 以此类推。在装配步
骤中利用Python建立集合, 每一个网格建立为一个单元集 合, 每一个网格待加工表面的节点建立为一个节点集合。 简化力在同一母线位置遵从可展直纹面的力添加规律, 即 载荷在同一母线 Vi上力的方向一致, 则需要在母线中点作 参考坐标系, 使得每一层载荷沿局部坐标系添加。在铣削 力的添加过程中, 利用UG作母线中点的参考坐标系获得 参考坐标系空间坐标, 在ABAQUS中利用三点法做出。对 叶片轴盘接触面进行完全约束, 模拟叶片固定在叶轮上的 效果, 不考虑装夹带来的影响, 效果图如图7所示。将 ABAQUS生成的inp文件中相对应的坐标点的空间坐标提 取出来在MATLAB中算取铣削力, 根据图 5 和图 6 利用 Python在每一个网格的待加工表面添加计算出来的铣削 力, 再利用生死单元法使切屑掉落。

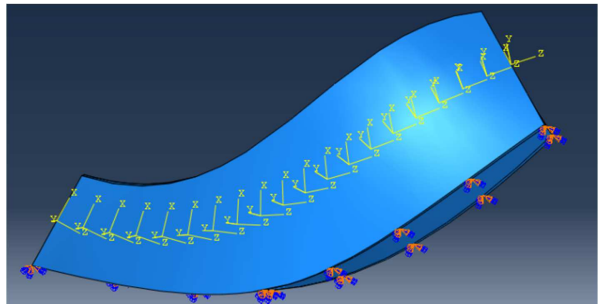

图7 添加边界条件和参考坐标系。

\section{2. 仿真过程及结果分析}

图7为加工某时刻的叶片应力分布, 以及让刀位移。

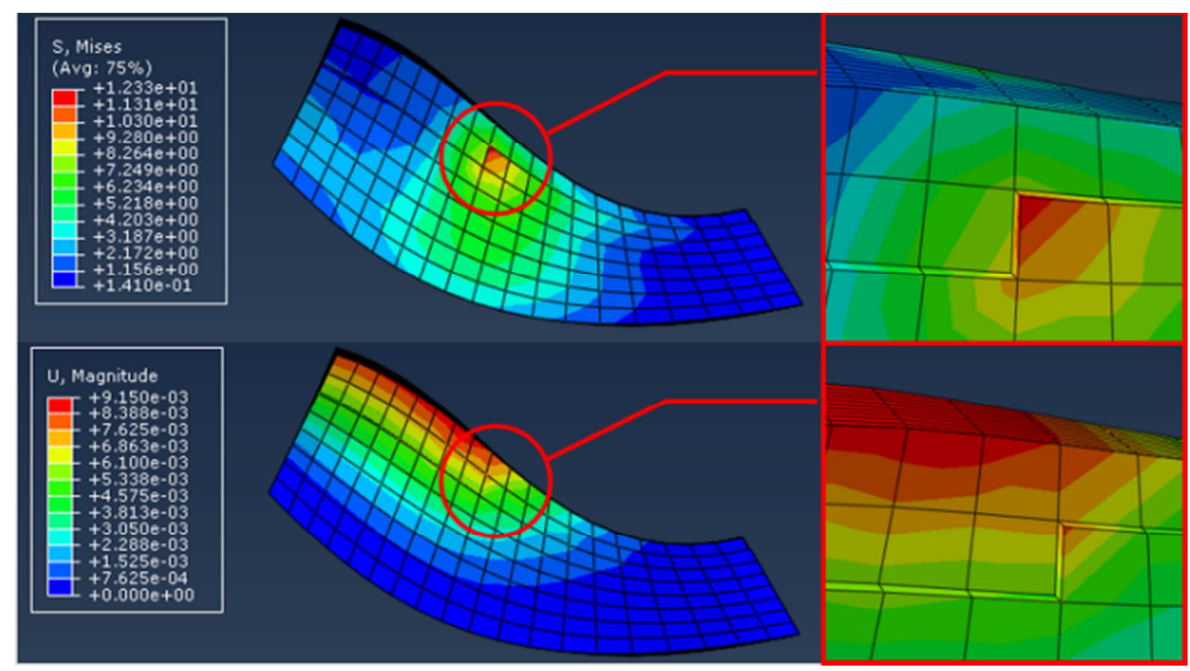

图8 单向铣削加工应力分布及位移分布。

铣削力的理论峰值出现在铣削刃与工件进行接触的 瞬间, 此时瞬时铣削厚度最大, 导致薄壁件的弹性让刀最 大。提取叶片在力加载瞬间的位移进行比较, 图9为单向 铁削过程中压力面的局部变形量。

比较不同加工层数可知, 在精加工过程中, 悬长量越 大, 弹性让刀越明显, 远端更能决定叶片加工质量, 因此 在研究让刀变形过程中可以单独研究 $\mathrm{B}$ 面悬长量最大的一 层。在同一层加工路径中, 切入和切出时的变形量比中间 的变形量要大。

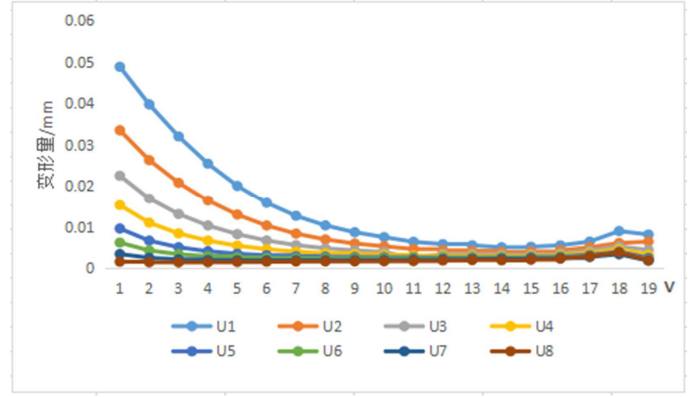

图9 单向铣削A面让刀变形。 
图10为单向加工和螺旋加工两种铣削顺序下吸力面B 的U1层的变形量, 由图可知, 螺旋铣削要比单向铣削的让 刀变形更小。这是由于在铣削B面U1层前, 单向铣削先铣

$\mathrm{A}$ 面整面, 螺旋铣削则是铣削 $\mathrm{A}$ 面 $\mathrm{U} 1$ 层单层, 导致工 件在螺旋铣削过程中整体刚度大于单向铣削, 因此让刀幅 值更低, 加工质量更好。由于单向铣削每层都是高点铣削 到低点, 而螺旋铣削为低点铣削到高点, 所以在低点处会 出现单向铣削质量略好的现象。

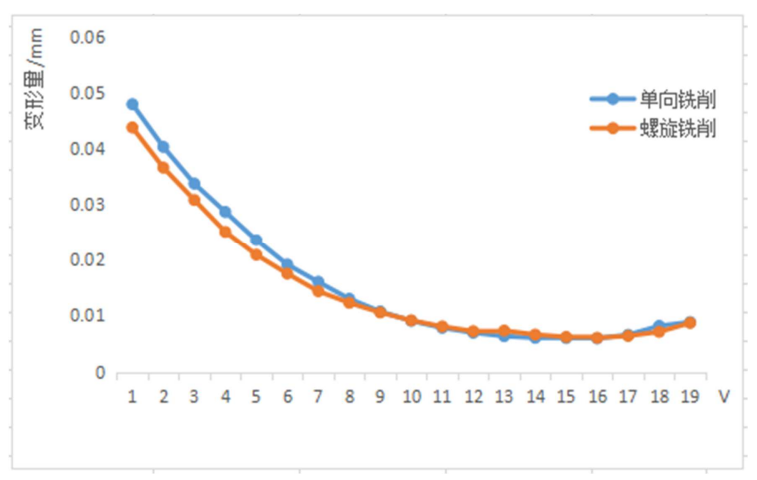

图10 吸力面 $\mathrm{B}$ 的 $\mathrm{U} 1$ 层让刀变形。

\section{5. 结论}

本文根据非均匀有理B样条原理在UG中对叶轮叶片 进行建模, 为了模拟铣刀在叶片上分层铣削的过程, 拟合 了钛合金的铣削力经验公式, 利用生死单元技术去除网格 以模拟铣削加工中的刚度变化。由仿真结果可知, 薄壁件 悬长量越大局部让刀变形越大, 螺旋铣削的加工顺序较单 向铣削在悬长量大的位置总刚度更大, 使得局部弹性变形 更小，加工误差更小，更适合进行叶片的精加工。

\section{参考文献}

[1] Caixu Yue, Zhitao Chen, Steven Y. Liang, Haining Gao, Xianli Liu. Modeling machining errors for thin-walled parts according to chip thickness $[\mathrm{J}]$. The International Journal of Advanced Manufacturing Technology, 2019, 103 (1-4): 91-100.

[2] 罗宇. 大型薄壁件加工变形预测及其影响因素研究[D].哈 尔滨：哈尔滨工业大学,2017.

[3] 钱丽丽. 钛合金薄壁件加工工艺分析与变形预测研究[D]. 南京: 南京航空航天大学,2013.
[4] 赵肖. 基于 “S”件精加工的铣削力精确建模和让刀变形预 测研究[D].成都: 电子科技大学, 2017 .

[5] S. Ratchev, S. Liu, A. A. Becker. Error compensation strategy in milling flexible thin-wall parts [J]. Journal of Materials Processing Tech., 2005, 162.

[6] S. Ratchev, S. Liu, W. Huang, A. A. Becker. An advanced FEA based force induced error compensation strategy in milling [J]. International Journal of Machine Tools and Manufacture, 2005, 46 (5).

[7] S. Ratchev, S. Liu, W. Huang, A. A. Becker. A flexible force model for end milling of low-rigidity parts [J]. Journal of Materials Processing Tech., 2004, 153-154.

[8] S. Ratchev, S. Liu, W. Huang, A. A. Becker. Milling error prediction and compensation in machining of low-rigidity parts [J]. International Journal of Machine Tools and Manufacture, 2004, 44 (15).

[9] S. Ratchev, W. Huang, S. Liu, A. A. Becker. Modelling and simulation environment for machining of low-rigidity components [J]. Journal of Materials Processing Tech., 2004, 153-154.

[10］王荣奇,谢雪范,周晓勤,李国发.薄壁结构件铣削加工变形的 有限元分析 [J].组合机床与自动化加工技术,2019(02):5-7.

[11] 袁俊松,孔啸,孙嘉继,李铭.铝合金薄壁件铣削加工变形有限 元分析[J].现代制造工程,2011(11):96-100.

[12] 于海滨. 直纹面型叶轮铣削变形及加工参数优化研究 [D]. 哈尔滨：哈尔滨理工大学,2015.

[13] 田燕宾. 半开式叶轮铣削加工变形及参数优化研究 [D].沈 阳: 东北大学,2015.

[14] 刘永峰. 基于有限元的叶片加工工艺参数确定方法研究[D]. 天津: 天津大学,2015.

[15] Shi Wu, Yangyang Wang, Xianli Liu, et al. Effects of the comprehensive stiffness performance of the impeller processing system on the machining error [J]. 2018, 13 (2): 151-167.

[16] 黄泽华. 整体叶轮铣削加工弹性变形预测及误差补偿研究 [D].北京交通大学,2013.

[17] 杨金龙. 钛合金整体叶盘侧铣加工刀具设计及性能评价分 析[D].哈尔滨: 哈尔滨理工大学,2019.

[18] 张以都, 张洪伟. 航空整体结构件加工变形有限元数值仿真 [J].北京航空航天大学学报,2009,35(02):188-192. 